Surge Capacity for Outbreak Investigations: A Partnership Between Columbia University and the New York City Department of Health and Mental Hygiene (NYC DOHMH)

S. Morse, ${ }^{1}$ M.M. McCollum, ${ }^{2}$ S.B. Balter, ${ }^{3}$ K.G. Gebbie, ${ }^{2}$ C.D. DiMaggio, ${ }^{2}$ E.G. Gebbie ${ }^{2}$

1 Columbia University Center for Public Health Preparedness, USA

2. Columbia University, USA

3. New York City Department of Health, USA

The Center for Public Health Preparedness (CPHP) at Columbia University has developed a training module for case investigation to provide surge capacity during emergencies. It has translated the model-aimed initially at public health workforce ancillary staff-to a new audience of student volunteers. During the pilot program in the summer of 2004, 12 students from Columbia's Mailman School of Public Health were given functional epidemiological training by CPHP faculty, including case investigation and interviewing skills. By doing this, the students provided surge capacity to the New York City Department of Health and Mental Hygiene (NYC DOHMH) to aid in the epidemiological response to a mass-casualty incident. This training was done using a module originally developed by CPHP for the Westchester County Department of Health and then carefully translated for an audience of public health students. These students were called upon several times during the summer to aid the NYC DOHMH during outbreaks of food-borne disease.

The NYC DOHMH found the partnership so effective that the program was expanded in the fall of 2004 to include $>30$ students from several departments in the Mailman School of Public Health. These volunteers are working currently with officials on outbreak investigations but also have been asked by the city to contribute to its public health education programs. Surveys of the student volunteer corps at the end of the 2004 summer training session and after each activation reveal that the training provided was extremely useful in the frontline practice of public health surveillance and disease investigation. The surveys further indicated a high interest from the students for continued participation in the partnership.

Infectious disease outbreaks are resource-intensive public health emergencies that often require the NYC DOHMH to reassign staff members in order to fill response teams necessary for participating in an investigation. Such investigations can disrupt the routine work of the health department. Recruiting student volunteer corps to assist with outbreaks and related public health surveillance could provide timely, effective resources for the city. In the event of large-scale, more serious public health emergencies, having students with prior, practical public health training could further augment the Department's surge capacity. For students, the partnership between the CPHP and the NYC DOHMH has strengthened their interest in public health practice by providing hands-on training and valuable frontline experience in the field. This allows student volunteers to become more familiar with public health trends in the city as well as health promotion and disease prevention initiatives that have succeeded in reaching desired target populations.

Keywords: New York; outbreak; public health; student volunteers; surge capacity

Prehosp Disast Med 2005;20(2):s73

\section{Mass-Casualty Incidents in Krakow Region, Poland}

P. Gula, ${ }^{1}$ W. Hladki, ${ }^{2}$ L. Brongel ${ }^{2}$

1. State Fire and Rescue Service Krakow, Poland

2. Emergency Medicine and Multiple Trauma Department, Medical College Jagiellonian University, Poland

The Krakow region is one of the major regions in Poland, with a population of $>3.2$ million people, and an area of $15,144 \mathrm{~km}^{2}$. The main city of the region is Krakow, with a population of one million. The authors analyzed masscasualty incidents (MCIs) that took place during a six-year period, from 01 January 1999-31 December 2004. Their characteristics are very similar to most $\mathrm{MCI}$ s taking place in Central European countries. Most of the MCIs could be defined as limited MCIs, in which the consolidation phase was achieved within less than one hour of incident duration. During this period, a total number of $28 \mathrm{MCIs}$ occurred (4.7 per year). Eight of them occurred in urban areas and 20 occurred in rural areas, with a distance of at least $15 \mathrm{~km}$ to the nearest hospital. The total number of injured requiring hospital treatment was 420 (15 per incident) with range of 10-37. The most common incidents were road-traffic crashes ( 20 incidents), among them 12 involved public transportation. Other cases included five fires and three chemical incidents. The authors will present procedures of cooperation between emergency services in MCIs on prehospital and hospital levels as well the most common problems.

Keywords: characteristics; mass-casualty incidents; Poland; prehospital

Prehosp Disast Med 2005;20(2):s73

\section{Hot Weather and the Emergency Room}

\section{J.L. Alonso; L. Higa Sansone; R. Martin Hierro; E. Lopez;}

\section{F. Farrais; $A$. Martin}

Hospital Universitario La Candelaria, Spain

During the summer of 2004, the Canary Islands suffered the most intense hot weather $\left(>42^{\circ} \mathrm{C}\right.$ with a mean of $27.5^{\circ} \mathrm{C}$ ) that meteorologists had ever recorded. This affected citizens and emergency rooms (ERs). The island's ERs were filled with patients with heat-related diseases. A study of these diseases, from heat stress to heat shock, will be presented.

All factors that contribute to heat shock such as age, living conditions, and disease, were studied. The following conclusions were made: (1) hot weather changes affects ERs; (2) elderly people affected by the heat had previous health problems; and (3) young people with heat shock were used to working in places with poor ventilation and/or high temperatures inside.

Keywords: Canary Islands; emergency room; hospital; heat-related illness

Prehosp Disast Med 2005;20(2):s73 